\title{
The flexible laryngeal mask airway in pediatric patients undergoing adenotonsillectomy
}

\begin{abstract}
Background and Aim: Endotracheal intubation is considered the premier airway device during adenotonsillectomies. Now-a-days flexible laryngeal mask airway is being used with an increased frequency during adenotonsillectomies. Aim of our study was to assess the feasibility of flexible laryngeal mask airway as a safer alternative to endotracheal tube in paediatric adenotonsillectomy.
\end{abstract}

Methods: Sixty children of 3-8 years of age group of either sex, weighing 10-20kg and belonging to physical status of ASAPS Grades 1 and 2 posted for elective adenotonsillectomy were studied. All patients were randomized into two groups. In group E appropriate sized endotracheal tube was used as the airway device. In group F-Flexible laryngeal mask airway (FLMA) size 2 was used. Haemodynamic responses during and following insertion of the devices were recorded. Incidence of cough, bronchospasm and laryngospasm were studied. The independent student's $t$ test and Chi-square test were used for the data analysis and a $\mathrm{p}$ value less than 0.05 was taken as significant.

Results: Our study revealed that the insertion as well as removal of FLMA was associated with fewer haemodynamic changes when compared with endotracheal tube. The Flexible LMA provided adequate surgical access, maintained adequate intraoperative oxygenation and ventilation and had lesser extubation time. The incidence of cough and laryngospasm was lower in patients where FLMA was used.

Conclusion: The Flexible LMA proves to be a suitable and safe alternative to endotracheal tube for airway management in elective, fasted, paediatric patients undergoing adenotonsillectomy.

Keywords: adenoidectomy, tonsillectomy, flexible, laryngeal mask airway
Volume 8 Issue 5 - 2017

\author{
Sagitha Joseph,' KoumudiVM, ${ }^{2}$ Shoba Philip ${ }^{3}$ \\ 'Department of Anaesthesiology, Junior Consultant, India \\ ${ }^{2}$ Anaesthesiology, Senior Consultant, Department of \\ Anaesthesiology, India \\ 3Anesthesiology, Senior Consultant and HOD, Department of \\ Anesthesiology, India
}

Correspondence: Sagitha Joseph, Department of

Anaesthesiology, Junior Consultant, Lourdes Hospital, Kochi I2, Kerala, India, Email sagithaj2000@gmail.com

Received: September 20, 2017 | Published: September 22, 2017

\section{Introduction}

Adenotonsillectomy is a unique challenging procedure for the anaesthesiologist as the airway is shared between the anaesthesia provider and the surgeon. Endotracheal intubation is the standard means to secure the airway in adenotonsillectomy. ${ }^{1}$ In recent years, the flexible laryngeal mask airway (FLMA) has been used with increased frequency as an alternative. ${ }^{2}$ This device has a longer, narrower, wire-reinforced airway tube, which gives the shaft more flexibility and resistance to compression. ${ }^{3-5}$ Use of Flexible LMA for adenotonsillectomy is widespread in Canada and Europe. Concern for aspiration of blood and secretions and obstruction of surgical field have limited the widespread use of the FLMA for adenotonsillectomy in our country. Hence the present study was undertaken with an aim to assess the safety profile and efficacy of FLMA for paediatric adenotonsillectomy.

\section{Materials and methods}

After obtaining institutional ethical committee approval, this prospective, unicentric, randomized trial was conducted between July 2013 and March 2014. Sixty children of 3-8 years of age group of either sex, weighing 10-20kg and belonging to physical status of ASA PS Grade 1 and 2 posted for elective adenotonsillectomy were studied. Sample size was determined by the formula $n>Z 2 P Q / d 2$, where $Z$ is the confidence coefficient, $\mathrm{P}$ is the $\%$ incidence in the population $\mathrm{Q}=100-\mathrm{P}, \mathrm{d}=$ error of estimation $(\mathrm{P}=50 \%, \mathrm{Q}=50 \%, \mathrm{Z}=1.96$ (by taking confidence as $95 \%$ and $\mathrm{d}=13 \%$ ) substituting in the above formula, $\mathrm{n}>57$. Hence in the present study we selected the sample size as 60 .
Patient who had any of the following condition was excluded from the study-Body mass index (BMI) $>35$, craniofacial anomalies, upper respiratory tract infections, anticipated difficult airway, hiatus hernia, gastroesophageal diseases, cardiovascular or respiratory diseases, convulsion, full stomach, grade 4 tonsils (tonsils occupy the entire transverse diameter of the oro pharynx). All patients were randomly allocated to one of the two groups of 30 patients each, using random number generator. In group $\mathrm{E}$ appropriate sized endotracheal tube was used as the airway device. In group F-Flexible LMA size 2 was used. A thorough pre-anaesthetic checkup was carried out and written informed consent was obtained from the parents. After confirming nil per oral status, all were premedicated with syrup promethazine $(0.5 \mathrm{mg} / \mathrm{kg})$ one hour prior to the induction of anaesthesia. In the operation theatre baseline parameters were recorded. Induction was done with inhalation of sevoflurane $8 \%$ and oxygen. Intravenous atropine $(0.02 \mathrm{mg} / \mathrm{kg})$ and fentanyl $(2 \mathrm{microgram} / \mathrm{kg})$ were given. Nasal endoscopy was done by the surgeon to assess the site of obstruction and to plan the surgery. Propofol $(2.5-3 \mathrm{mg} / \mathrm{kg})$ was then given to facilitate the insertion of the airway device. After ensuring adequate depth of anaesthesia ETT or FLMA was inserted. All the airway insertions were done by an anaesthesiologist with an experience of more than 5 years. Endtidal $\mathrm{CO}_{2}$ monitor was connected and ventilated using Jackson Rees modification of Ayre's T piece. The position of endotracheal tube (ETT) or FLMA was checked by observing the movements of chest wall, auscultation of breath sounds and absence of audible leak during controlled ventilation. Muscle relaxant atracurium $(0.5 \mathrm{mg} / \mathrm{kg})$ was given to maintain the airway device and intermittent boluses of atracuriun $0.1 \mathrm{mg} / \mathrm{kg}$ were given as required. 
Monitoring of the patient was done throughout the procedure as per ASA standards. The ventilation was controlled to maintain a target $\mathrm{SpO}_{2}(>95 \%)$ and $\mathrm{EtCO}_{2}(35-45 \mathrm{~mm}$ of $\mathrm{Hg})$. Patient was positioned supine with a shoulder roll to achieve extension of the neck. A Boyle Davis mouth gag was inserted and opened gradually, while carefully monitoring breath sounds and capnograph. Displacement of the device was suspected if there was a change in the ease of ventilation or an audible leak in the pharynx was noticed. In such a situation repositioning of the FLMA and reapplication of the gag was done. All the surgeries were performed by an experienced paediatric otolaryngologist who was familiar with the use of FLMA. Adenoidectomy was done using suction diathermy and tonsillectomy using a microdebrider.

Anaesthesia was by sevoflurane (2-3\%), nitrous oxide $50 \%$ and oxygen $50 \%$. Diclofenac suppository $(2 \mathrm{mg} / \mathrm{kg})$ was kept rectally for post-operative analgesia. Intraoperatively dexamethasone $(0.2 \mathrm{mg} /$ $\mathrm{kg}$ ) and ondansetron $(0.1 \mathrm{mg} / \mathrm{kg})$ were given intravenously to prevent postoperative nausea and vomiting. After the completion of surgical procedure all anaesthetic agents were discontinued and the residual neuromuscular blockade was reversed with neostigmine $(0.05 \mathrm{mg} / \mathrm{kg})$ and glycopyrrolate $(0.01 \mathrm{mg} / \mathrm{kg})$. Once child was fully awake, opened eyes on command and adequate tidal volume achieved, the airway device was removed after deflating the cuff in a head down and left lateral decubitus position. Patients were monitored throughout the perioperative period including the stay in the postanaesthesia care unit. Haemodynamic responses (heart rate and arterial blood pressure) were recorded before induction, after induction (before airway device placement), 10 seconds, 1 minute, 3 minutes, 5 minutes after insertion of the airway device and at every 10 minutes interval thereafter. It was also noted 10seconds after removal of the device. Any episode of laryngospasm, bronchospasm and vomiting during insertion of device was recorded. Adequacy of surgical exposure was confirmed with the surgeon.

After extubation any episode of laryngospasm, persistent coughing (i.e, two or more episode of stressful or violent coughing or Spo2 falling below 95\% during coughing episodes), vomiting and blood staining of the airway device (ie, inside the glottis portion of ETT and undersurface of LMA cuff) were noted to evaluate the laryngopharyngeal morbidity produced. Extubation time (ie, time in minutes from surgery end to extubation) was recorded. All the data was collected by a senior resident and was then subjected to statistical

Table I Demographic and baseline clinical data analysis. The following tests were used and a $p$ value of less than 0.05 was taken as significant. Independent sample ' $t$ ' test for comparison of the means of the two groups with respect to the age, weight, height, BMI, haemodynamic responses (heart rate \& mean arterial blood pressure) and extubation time. Chi-square test for the comparison of sex, ASA PS Grade and the incidence of laryngopharyngeal morbidities.

\section{Results}

Children who underwent adenotonsillectomy between July 2013 and March 2014 were recruited for the study. The physical characteristics of patients were comparable in both the groups (Table 1). The heart rate and mean arterial pressure increased significantly in both groups after insertion of ETT/FLMA as shown in Table 2. The percentage rise in mean heart rate and mean blood pressure from preinsertion to 10 seconds, 1 minute, 3 minutes, 5 minutes after device placement was significantly higher in the ETT group as compared to FLMA group $(\mathrm{p}<0.05)$ as shown in Table $3 \& 4$. The percentage rise in mean hear trate and mean blood pressure from before removal to 10 seconds after removal were also significantly higher in the ETT group $(p<0.05)$ whereas after removal of FLMA there was not much increase in both. Analysis of the trend in haemodynamics showed that the change in mean heart rate and mean arterial pressure from preinsertion levels persisted for more time in the ETT group and came back to preinsertion value after 5 minutes whereas in the FLMA group it came to preinsertion value at 1 minute.

Patients in both groups maintained adequate oxygenation and ventilation in the perioperative period and there was no incidence of aspiration in either group. The surgical exposure was adequate in all the cases. The extubation time (time from the end of the surgery to extubation) was measured and the mean extubation time was 10 minutes and 40 seconds in the ETT group and 5 minutes and 42 seconds in the FLMA group (Figure 1) and there was a difference of 4 minutes and 41 seconds and it was found to be statistically significant (p-0.00). None of the patients studied had any incidence of laryngospasm, bronchospasm and vomiting on placement of the airway device. Further the incidence of laryngospasm $(3 \%$ in group $\mathrm{E}$ and $0 \%$ in group $\mathrm{F}$ ) and cough on removal of the device (20\% in group $\mathrm{E}$ and $7 \%$ in group F) was lower in the flexible LMA group, but the difference was not statistically significant. After removal, the device was inspected for blood staining and none of the device showed the same (Table 5) (Figure $2 \& 3$ ).

\begin{tabular}{llll}
\hline Parameters & ETT Group $(\mathbf{n}=\mathbf{3 0})$ & FLMA Group $(\mathbf{n}=\mathbf{3 0})$ & P value \\
\hline Age in years & $5.80+1.064$ & $5.37+1.273$ & 0.158 \\
Sex & 19 males \& II females & 17 males \& I3 females & 0.598 \\
BMI $(\mathrm{Kg} / \mathrm{m} 2)$ & $17.13+1.98 \mid$ & $17.87+2.315$ & 0.19 \\
ASA PS Grade & 25 (grade I) \& 5(grade 2) & $27($ grade I) \& 3(grade 2) & 0.448 \\
Baseline heart rate(beats $/ \mathrm{min})$ & $83.2+4.852$ & $81.30+4.843$ & 0.134 \\
Baseline MAP $(\mathrm{mm} \mathrm{Hg})$ & $56.73+3.97$ & $58.53+4.24$ & 0.095 \\
\hline
\end{tabular}

Table 2 Trend of haemodynamic responses

\begin{tabular}{|c|c|c|c|c|c|c|c|c|}
\hline & Baseline & Preinsertion & $\begin{array}{l}\text { I0 s After Device } \\
\text { Placement }\end{array}$ & $\begin{array}{l}\text { I min After } \\
\text { Placement }\end{array}$ & $\begin{array}{l}\text { 3mn After } \\
\text { Placement }\end{array}$ & $\begin{array}{l}\text { 5mn After } \\
\text { Placement }\end{array}$ & $\begin{array}{l}\text { Before } \\
\text { Removal }\end{array}$ & $\begin{array}{l}\text { I0 s After } \\
\text { Removal }\end{array}$ \\
\hline \multicolumn{9}{|l|}{ Group E } \\
\hline Heart Rate(beats/min) & $83.2 \pm 4.85$ & $120.8 \pm 3.69$ & $134.9 \pm 3.96$ & $132.1 \pm 3.68$ & $128.9 \pm 3.66$ & $124.3 \pm 3.42$ & $124.7 \pm 3.16$ & $|3| . \mid . \pm 3.63$ \\
\hline $\begin{array}{l}\text { Mean Arterial } \\
\text { Pressure }(\mathrm{mmHg})\end{array}$ & $56.7 \pm 3.97$ & $59 \pm 3.75$ & $69.9 \pm 3.27$ & $66.7 \pm 3.01$ & $63.9 \pm 3.47$ & $61.2 \pm 3.67$ & $57.5 \pm 3.19$ & $61.6 \pm 3.30$ \\
\hline \multicolumn{9}{|l|}{ Group F } \\
\hline Heart Rate & $81.3 \pm 4.84$ & $119.5 \pm 5.10$ & $121.1 \pm 5.17$ & $119.6 \pm 5.8$ & $117.7 \pm 6.69$ & $115.7 \pm 6.73$ & $118.6 \pm 5.93$ & $119.6 \pm 6.14$ \\
\hline $\begin{array}{l}\text { Mean Arterial } \\
\text { Pressure }\end{array}$ & $58.5 \pm 4.25$ & $60.1 \pm 4.89$ & $61.5 \pm 4.95$ & $60.3 \pm 4.67$ & $58.6 \pm 4.97$ & $57.1 \pm 4.88$ & $53.6 \pm 4.68$ & $55.1 \pm 4.78$ \\
\hline
\end{tabular}


Table 3 Comparison of mean percentage rise in heart rate

\begin{tabular}{|c|c|c|c|c|c|c|c|}
\hline & Group & Mean & SD & Mean Difference & t - value & df & $p$ - value \\
\hline \multirow{2}{*}{ Pre insertion HR vs $H R$ at I0 seconds } & ETT & 11.66 & 1.493 & \multirow{2}{*}{10.31} & \multirow{2}{*}{29.087} & \multirow{2}{*}{58} & \multirow{2}{*}{0} \\
\hline & FLMA & 1.35 & $1.24 \mid$ & & & & \\
\hline \multirow{2}{*}{ Pre insertion HR vs HR Imin } & ETT & 9.37 & 1.509 & \multirow{2}{*}{9.298} & \multirow{2}{*}{23.047} & \multirow{2}{*}{58} & \multirow{2}{*}{0} \\
\hline & FLMA & 0.07 & 1.615 & & & & \\
\hline \multirow{2}{*}{ Pre insertion HR vs $H R$ at 3 min } & ETT & 6.67 & 1.738 & \multirow{2}{*}{8.212} & \multirow{2}{*}{13.76} & \multirow{2}{*}{58} & \multirow{2}{*}{0} \\
\hline & FLMA & -1.54 & 2.769 & & & & \\
\hline \multirow{2}{*}{ Pre insertion $H R$ vs $H R$ at 5 min } & ETT & 2.89 & 1.4 & \multirow{2}{*}{6.139} & \multirow{2}{*}{10.863} & \multirow{2}{*}{58} & \multirow{2}{*}{0} \\
\hline & FLMA & -3.25 & 2.76 & & & & \\
\hline \multirow{2}{*}{ HR before removal vs HR I0s after removal } & ETT & 4.77 & 1.111 & \multirow{2}{*}{3.949} & \multirow{2}{*}{15.805} & \multirow{2}{*}{58} & \multirow{2}{*}{0} \\
\hline & FLMA & 0.82 & 0.8 & & & & \\
\hline
\end{tabular}

Table 4 Comparison of mean percentage rise in MAP

\begin{tabular}{|c|c|c|c|c|c|c|c|}
\hline & Group & Mean & SD & Mean Difference & t - value & df & $p$ - value \\
\hline \multirow{2}{*}{ Preinsertion MAP vs MAPat I0 seconds } & ETT & 18.67 & 2.768 & \multirow{2}{*}{16.314} & \multirow{2}{*}{25.602} & \multirow{2}{*}{58} & \multirow{2}{*}{0} \\
\hline & FLMA & 2.36 & 2.126 & & & & \\
\hline \multirow{2}{*}{ Pre insertion MAP vs MAP at I min } & ETT & 13.2 & $2.84 I$ & \multirow{2}{*}{12.867} & \multirow{2}{*}{19.746} & \multirow{2}{*}{58} & \multirow{2}{*}{0} \\
\hline & FLMA & 0.33 & 2.16 & & & & \\
\hline \multirow{2}{*}{ PreinsertionMAPvs MAP at 3 mins } & ETT & 8.39 & 2.307 & \multirow{2}{*}{$|0.88|$} & \multirow{2}{*}{17.313} & \multirow{2}{*}{58} & \multirow{2}{*}{0} \\
\hline & FLMA & -2.49 & 2.555 & & & & \\
\hline \multirow{2}{*}{ Pre insertion MAP vs MAP at $5 \mathrm{mins}$} & ETT & 3.72 & 2.333 & \multirow{2}{*}{8.704} & \multirow{2}{*}{12.827} & \multirow{2}{*}{58} & \multirow{2}{*}{0} \\
\hline & FLMA & -4.98 & 2.893 & & & & \\
\hline \multirow{2}{*}{ MAP before removal vs MAP IOs after removal } & ETT & 5.89 & 1.801 & \multirow{2}{*}{3.498} & \multirow{2}{*}{8.211} & \multirow{2}{*}{58} & \multirow{2}{*}{0} \\
\hline & FLMA & 2.39 & 1.484 & & & & \\
\hline
\end{tabular}

Table 5 Incidence of laryngopharyngeal morbidities

\begin{tabular}{llll}
\hline Laryngopharyngeal Morbidities & Group E & Group F & P value \\
\hline Laryngospasm on insertion & $\mathrm{Nil}$ & $\mathrm{Nil}$ & - \\
Bronchospasm on insertion & $\mathrm{Nil}$ & $\mathrm{Nil}$ & - \\
Vomiting on insertion & $\mathrm{Nil}$ & $\mathrm{Nil}$ & - \\
Laryngospasm after removal & $3 \%$ & $\mathrm{Nil}$ & 0.999 \\
Coughing after removal & $20 \%$ & $7 \%$ & 0.254 \\
Bloodstaining of the device & $\mathrm{Nil}$ & $\mathrm{Nil}$ & - \\
\hline
\end{tabular}

\section{Discussion}

Laryngeal mask airway was introduced in UK in 1983 by AIJ Brain. ${ }^{6}$ Over the years it has evolved as a primary airway device and has replaced endotracheal tube in several situations.One such surgery is adenotonsillectomy. The FLMA can achieve most of the requirements of a surgeon and an anaesthesiologist. ${ }^{7}$ Using a reinforced tube (flexible LMA) can prevent the kinking caused by the mouth gag. When FLMA is in place, it completely covers the glottis and hence doesn't allow any soiling of the airway. The use of FLMA decreases the requirement of muscle relaxation and hence ensures speedy discharges. ${ }^{8}$ Decreased incidence of cough and laryngospasm are other major benefits of LMA. ${ }^{9-13}$ Another advantage of LMA placement over endotracheal intubation is the minimal disturbances in cardiovascular and respiratory system at insertion and removal. ${ }^{14}$ Several studies done in paediatric patients comparing the haemodynamic responses during placement of LMA and an endotracheal tube concluded that haemodynamic response was less and was short lived with laryngeal mask airway as compared to endotracheal intubation (Jamil SN et al. ${ }^{9}$, SN Hufeza et al. ${ }^{15}$, Thomas $\mathrm{R}$ et al. ${ }^{16}$, Lalwani $\mathrm{J}$ et al. ${ }^{17}$ ). We also had a similliar observation. There was minimum stress response with Flexible LMA placement when compared with endotracheal intubation. The increase in haemodynamic parameters during removal was more in the ETT group when compared to FLMA group. The haemoglobin oxygen saturation was monitored throughout and it never dropped below $99 \%$ in both the groups. The end tidal $\mathrm{CO}_{2}$ concentration was maintained in a range of $35-40 \mathrm{~mm} \mathrm{Hg}$. None of the children in the FLMA group had a problem that required replacement with an endotracheal tube which proved that FLMA was effective in maintaining adequate oxygenation and ventilation perioperatively. Similar results were obtained by SN Jamil et al. ${ }^{9}$

All cases had adequate surgical exposure,as confirmed by the surgeon. Peng A et al., ${ }^{8}$ observed the surgical exposure in adenotonsillectomy while using FLMA and found that it provided adequate surgical access in $93.8 \%$ of patients. Aziz $\mathrm{L}^{18}$ also had reported adequate surgical access where FLMA was used. The extubation time was measured and there was a difference of 4 minutes and 41 seconds and it was found to be statistically significant. This is similar to the observation by A Peng et al. ${ }^{8}$ where they compared the extubation time among paediatric adenotonsillectomies and observed a significantly shorter time by 4.06 minutes for patients where FLMA was used as the airway device. None of the patients in our study had an incidence of laryngospam, bronchospam and vomiting on placement of the airway device. The incidence of laryngospasm on removal of the device was found to be more in the ETT group (ETT-3.3\%, FLMA- nil). However the difference was not statistically significant $(\mathrm{p}>0.05)$. Laryngospasm was relieved by positive pressure ventilation. Pankaj Kundra et al., ${ }^{19}$ had reported an incidence of laryngospasm as 9\% in the ETT group where as it was nil in the FLMA group. The 
incidence of cough after removal was $20 \%$ in the ETT group where as it was only $6.7 \%$ in the FLMA group in our study. However the difference was not statistically significant ( $p$ value was 0.254 ). Similar findings were observed in other studies also. Ali CA et al., ${ }^{2}$ found that there was more coughing after extubation (ETT-17\%, FLMA-5.7\%), than after withdrawal of the FLMA. A study by DI Sierpina et al., ${ }^{20}$ demonstrated that, ETT use was associated with more coughing $(48 \%$ for ETT and 20\% for FLMA). Punkaj Kundra et al., ${ }^{19}$ had reported an increased incidence of persistent cough in the ETT group (36\% ETT vs $15 \%$ FLMA).

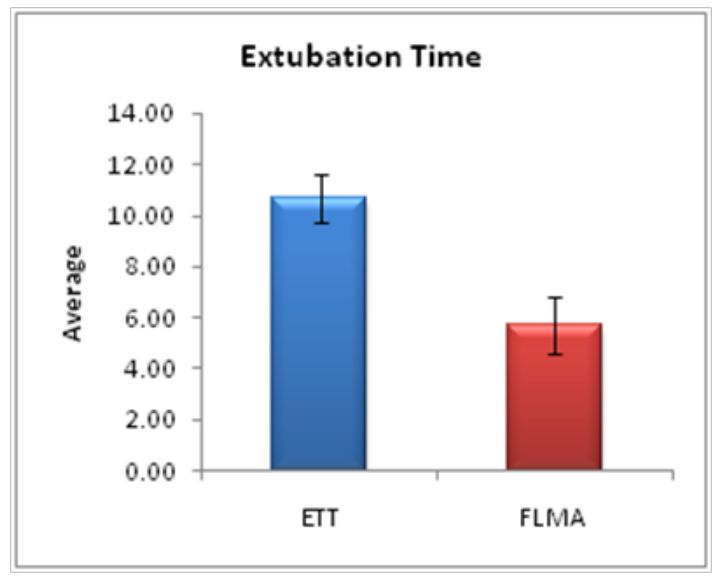

Figure I Mean extubation time in minutes.

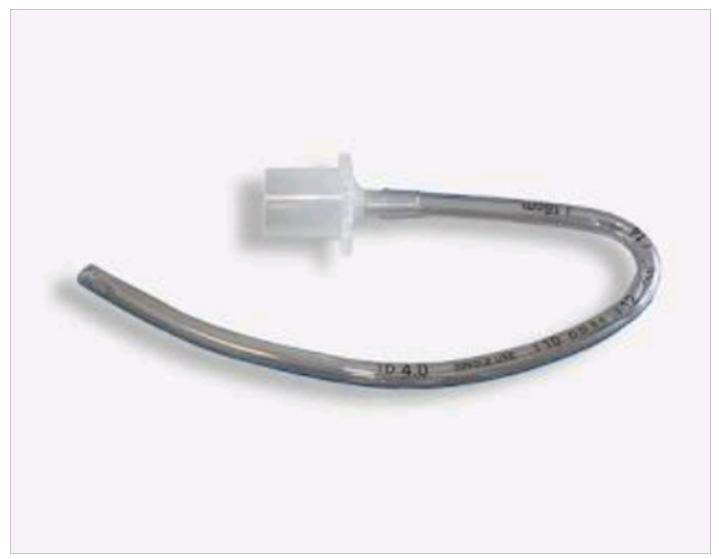

Figure 2



Figure 3
In our study both the devices were inspected for blood staining (surface of the ETT introduced beyond the glottic opening and the laryngeal aspect of the FLMA) and none of the devices had showed the same. Hence it was concluded that FLMA provided adequate protection of the airway. This could be due to the surgical position of the hyperextended neck for adenotonsillectomy which keeps the oropharynx at a lower level than the glottis thereby preventing the trickling of blood into the trachea and the surgical technique of our surgeon. Amongst various studies evaluating blood staining of the airway device, the study by A Peng ${ }^{8}$ performed a flexible fiberoptic bronchoscopy through the FLMA at the conclusion of adenotonsillectomy and blood was noted in the laryngeal inlet in only 1 case. Our study had certain limitations. A fiberoptic intubating device could be used for confirmation of the position of FLMA and also to assess the presence of blood in the larynx. Seal pressures of the FLMA could be studied to further evaluate the safety profile of the airway device. All surgeries were performed by an experienced paediatric otolaryngologist making our results less applicable to centers at which these conditions do not apply. It would be better if the intubation was done using muscle relaxant which would have reduced the haemodynamic responses.

\section{Conclusion}

Our study revealed that the insertion as well as removal of FLMA was associated with fewer haemodynamic changes when compared with endotracheal tube. The Flexible LMA further provided adequate surgical access, maintained adequate intraoperative oxygenation and ventilation and had lesser extubation time. With regards to the laryngopharyngeal morbidity our study found that the incidence of laryngospasm and cough were lower in patients subjected to FLMA placement. Hence we conclude that the Flexible LMA proves to be a suitable and safe alternative to endotracheal tube for airway management in elective, fasted, paediatric patients undergoing adenotonsillectomy.

\section{Acknowledgments}

None.

\section{Conflicts of interest}

Authors declare that there is no conflict of interest.

\section{References}

1. Lalwani K, Richins S, Aliason I, et al. The laryngeal mask airway for pediatric adenotonsillectomy: Predictors of failure and complications. Int J Pediatr Otorhinolaryngol. 2013;77(1):25-28.

2. Ali CA, Imam SM, Anwar K, et al. Comparison of the reinforced laryngeal mask airway and endotracheal tube intubation in adult tonsillectomy. Gomal J Med Sci. 2014;12(1):2-6.

3. Sood J. Laryngeal mask airway and its variants. Indian J Anaesth. 2005;49(4):275-280.

4. Dorsch JA, Dorsch SE. Supraglottic Airway Devices. In: Understanding Anesthesia Equipment. 5th ed. Williams and Wilkins: New Delhi, India; 2008. 461-491, 553-554 p.

5. Patel A. LMA Flexible A Practical Guide. The Laryngeal Mask Company Limited; 2006.

6. Brain AIJ. The laryngeal mask airway a new concept in airway management. Br J Anaesth. 1983;55(8):801-805.

7. Raafat S Hannallah, Karen A Brown, Susan $\mathrm{T}$ Verghese. Otorhinolaryngologic Procedures. In: Cote CJ, et al. (Eds.), A Practice of Anaesthesia for Infants and Children. 5th ed. Saunders, Philadelphia, USA; 2009. 660-671 p. 
8. Peng A, Dodson KM, Thacker LR, et al. Use of laryngeal mask airway in pediatric adenotonsillectomy. Arch Otolaryngol Head Neck Surg. 2011;137(1):42-46.

9. Jamil SN, Alam M, Usmani H, et al. A study of the use of laryngeal mask airway in children and its comparison with endotracheal intubation. Indian J Anaesth. 2009;53(2):174-178.

10. Goyal R. Small is the new big: An overview of newer supraglottic airways for children. J Anaesthesiol Clin Pharmacol. 2015;31(4):440-449.

11. Caughlin BP, Bhushan B, Maddalozzo J. Laryngeal Mask Airway versus Endotracheal Tube Intubation for Repairing of Nasal bone Fracture: A 7 Year Single Institution Case Control Study. Surgery Curr Res. 2015;5:223.

12. Abiruchi Patki. Laryngeal mask airway vs the endotracheal tube in paediatric airway management: A meta-analysis of prospective randomized controlled trials. Indian J Anaesth. 2011;55(5):537-541.

13. Suvarna D, Fernandes S. Anaesthesia Management of Adenotonsillectomy. Int J Otorhinolaryngol Clin. 2015;7:17-21.

14. Patel MG, Swadia VN, Bansal G. Prospective randomized comparative study of use of PLMA and ET tube for airway management in children under general anaesthesia. Indian J Anaesth. 2010;54(2):109-115.
15. Huzefa SN, Kamath SS, Misquith J. A comparative study of the effects of placement of laryngeal mask airway Vs endotracheal tube on haemodynamic parameters in children. Res Rev J Med Health Sci. 2013;3:61-66

16. Thomas R, Rahaman HAA, Padmanabh S. Prospective comparative study of haemodynamic changes during insertion of laryngeal mask airway versus endotracheal tube in paediatric patients. $J$ Evid Based Med Health. 2016;3:2035-2038.

17. Lalwani J, Dubey KP, Sahu BS, et al. ProSeal laryngeal mask airway: An alternative to endotracheal intubation in paediatric patients for short duration surgical procedures. Indian J Anaesth . 2010;54(6):541-545.

18. Aziz L, Bashir K. Comparison of armoured laryngeal mask airway with endotracheal tube for adenotonsillectomy. J Coll Physicians Surg Pak. 2006;16(11):685-688.

19. Kundra P, Supraja N, Agrawal K, et al. Flexible laryngeal mask airway for cleft palate surgery in children: a randomized clinical trial on Efficacy and Safety. Cleft Palate Craniofac J. 2009;46(4):368-373.

20. Sierpina DI, Chaudhary H, Walner DL, et al. Laryngeal mask airway versus endotracheal tube in pediatric adenotonsillectomy. Laryngoscope. 2012;122(2):429-435. 